\title{
TOXICIDADE AGUDA DE HERBICIDA E DE SEUS COMPONENTES QUIIMICOS DIURON E HEXAZINONA EM CERIODAPHNIA DUBIA
}

\section{Acute toxicity of herbicide and its components in chemical diuron and hexazinone in Ceriodaphnia dubia}

\author{
Bruno Moreira da Silva', Cristina Filomêna Pereira Rosa Paschoalato², Marcia Maisa de Freitas Afonso³, \\ Maira Batista de Souza ${ }^{4}$
}

Recebido em 07 de novembro de 2013; recebido para revisão em 05 de dezembro de 2013; aceito em 10 de dezembro de 2013; disponível on-line em 13 de dezembro de 2013.

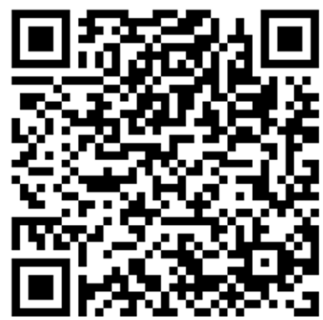

PALAVRAS CHAVES:

Ceriodaphnia dubia;

Diuron;

Ecotoxicidade aguda;

Hexazinona.

KEYWORDS:

Acute ecotoxicity;

Ceriodaphnia dubia;

Diuron;

Hexazinon.

\begin{abstract}
RESUMO: A expansão da cultura da cana-de-açúcar, a necessidade do uso de herbicidas, o processo de escoamento superficial da água no solo, a simplicidade das tecnologias empregadas nas estações de tratamento de água, justificam a proposta do referido estudo para avaliação dos efeitos ecotoxicológicos de um herbicida comercial composto por Diuron $(46,8 \% \mathrm{p} / \mathrm{p})$ e Hexazinona $(13,2 \% \mathrm{p} / \mathrm{p})$ e dos compostos puros Diuron e Hexazinona, através da realização de testes de toxicidade aguda, utilizando como organismo testes a Ceriodapnhia dubia. A metodologia utilizada nos ensaios foi adaptada das Normas NBR 12.713 (ABNT, 2004) e NBR 13.373 (ABNT, 2005). Nos ensaios de toxicidade aguda, o tempo de exposição foi de $48 \mathrm{~h}$ e os resultados obtidos foram estatisticamente processados por Trimmed Spearman-Karber para cálculo die $50 \%$ da imobilização dos organismos testes $\left(\mathrm{CE}_{50}\right)$. $\mathrm{O}$ valor obtido de $\mathrm{CE}_{50}$ para os testes agudos com um herbicida comercial foi de $5,78 \mathrm{mg} \cdot \mathrm{L}^{-1}$, para o Diuron o valor de $\mathrm{CE}_{50}$ foi de $2,9 \mathrm{mg} \cdot \mathrm{L}^{-1}$ e para Hexazinona o $\mathrm{CE}_{50}$ foi de $105,56 \mathrm{mg} \cdot \mathrm{L}^{-1}$. Concluiu-se que Diuron apresentou potencial tóxico de aproximadamente 37 vezes maior, quando comparado a Hexazinona e em relação aos valores obtidos de toxicidade citados na literatura, mesmo considerando organismos pertencentes a diferentes cadeias tróficas, os valores de $\mathrm{CE}_{50}$ apresentaram-se próximos, porém, faz se necessário a compilação de diversos ensaios em diferentes níveis tróficos, no intuito de ampliar dados para futura análise de risco que possibilitem aos órgãos regulamentadores o estabelecimento de limites máximos permissíveis para qualidade de águas superficiais com segurança e confiabilidade.
\end{abstract}

ABSTRACT: The expansion of the culture of cane sugar, the need for the use of herbicides, the process of runoff water in the soil, the simplicity of the technologies used in water treatment plants, justifies the proposal of this study to evaluate the effects ecotoxicological effects of a commercial herbicide Diuron consisting of $(46.8 \% \mathrm{w} / \mathrm{w})$ and Hexazinone $(13.2 \% \mathrm{w} / \mathrm{w})$ pure compounds and Diuron and Hexazinone, by carrying out acute toxicity tests using as test organism to Ceriodapnhia dubia. The methodology used in the tests was adapted from the NBR 12713 (ABNT, 2004) and NBR 13373 (ABNT, 2005). In acute toxicity tests, the exposure time was $48 \mathrm{~h}$ and the results obtained were statistically processed by Trimmed Spearman-Karber calculation to $5.0 \%$ of the test organisms immobilization $\left(\mathrm{EC}_{50}\right)$. The $\mathrm{EC}_{50}$ value obtained for the acutte tests with a commercial herbicide was $5.78 \mathrm{mg} \cdot \mathrm{L}^{-1}$ for Diuron the $\mathrm{EC}_{50}$ value was $2.9 \mathrm{mg} \cdot \mathrm{L}^{-1}$ and the $\mathrm{EC}_{50}$ was Hexazinone $105.56 \mathrm{mg} \cdot \mathrm{L}^{-1}$.Diuron was concluded that showed toxic potential of approximately 37- fold higher as compared to Hexazinone and for the toxicity values reported in literature, even considering organisms belonging; to different trophic chains, the $\mathrm{EC}_{50}$ values were close, but makes it necessary to buill several tests at different trophic levels, in order to expand data for future risk analysis that enable regulators to establish maximum allowable limits for surface water quality with safety and reliability.

* Contato com o autor:

1 e-mail : brunomoreira.eng@gmail.com (B. M. da Silva)

Aluno de iniciação científica curso de Engenharia Química, Universidade de Ribeirão Preto (UNAERP).

2e-mail : cpaschoa@gunaerp.br (C. F. P. R. Paschoalato)

Engenheira Química, Doutora em Engenharia Civil, Docente do programa de Pós Graduação em Tecnologia Ambiental da UNAERP.

3 e-mail : mafonso@unaerp.br (M. M. F. Afonso)

Engenheira Química, Doutora em Química Orgânica, Docente do Curso de Engenharia Química da UNAERP

${ }^{4}$ e-mail : brunomoreira.eng@gmail.com (M. B. de Souza)

Mestre em Tecnologia Ambiental pela UNAERP. 


\section{INTRODUÇÃO}

O cultivo da cana tornou-se um grande investimento agrícola e atualmente a cana-deaçúcar ocupa cerca de $2 \%$ da área cultivável no Brasil, que é o maior produtor mundial, seguido pela Índia, Tailândia e Austrália. No Brasil, as regiões que se destacam no cultivo da cana-deaçúcar são: Sudeste, sendo São Paulo o estado de maior índice seguido de Minas Gerais, Centro-Oeste (com Mato Grosso do Sul e Goiás), Sul e Nordeste. Em média $55 \%$ da colheita de cana-de-açúcar é destinada a fabricação de etanol e $45 \%$ para a fabricação de açúcar (UNICA, 2013).

A área cultivada de cana-de-açúcar destinada à atividade sucroalcooleira está estimada em 9,62 milhões de hectares, sendo $13 \%$ na região norte-nordeste e $87 \%$ na região centro-sul com 7,8 milhões de hectares para a safra 2013 (UNICA, 2013). A produtividade média da cultura de cana de açúcar no Brasil está estimada em 69289 kg/ha (CONAB, 2011). Com o propósito de aumento na produtividade agrícola são necessários e indispensáveis investimentos em agroquímicos e insumos, tais como os fertilizantes, formicidas, pesticidas e, principalmente, os herbicidas.

O uso de agroquímicos é a segunda causa de contaminação da água no país, só perde para o despejo de esgoto doméstico. A poluição da água provocada por agrotóxico ou fertilizante é um problema para $16,2 \%$ dos municípios brasileiros (901 municípios), na Bacia Costeira do Sul, 31\% dos municípios registraram poluição da água por agrotóxicos e $19 \%$ estão concentrados nas bacias do Rio da Prata e na Costeira do Sudeste (IBGE, 2010).

Dentre vários herbicidas empregados na cultura da cana-de-açúcar, um produto comercial composto pela mistura de Diuron e Hexazinona vem sendo amplamente utilizados (ARMAS, 2006).

A Resolução do Conselho Nacional do Meio Ambiente (CONAMA) $n^{\circ} 357$ (BRASIL, 2005), que estabelece padrões de qualidade para corpos d'água, não relaciona esses compostos químicos e recomenda que possíveis substâncias causadoras de danos aos seres vivos deverão ser investigadas, e que critérios de toxicidade deverão se basear em resultados de testes de ecotoxicológicos padronizados utilizando organismos aquáticos.

A ecotoxicologia é uma área especializada da toxicologia ambiental que analisa os efeitos tóxicos causados por agentes químicos e físicos sobre as populações em ambientes aquático e/ou terrestres, que integram o ecossistema. 0 desenvolvimento de protocolos para testes de toxicidade permitem definir a faixa de toxicidade tolerável obtendo-se o conhecimento de níveis aceitáveis e servem de referência na tomada de decisões para órgão públicos reguladores (RONCO et al., 2004).

A Portaria no 2914 do Ministério da Saúde (BRASIL, 2011), que estabelece padrões de potabilidade da água para consumo humano, incluiu e limitou o valor máximo de concentração de Diuron em $90 \mu \mathrm{g} . \mathrm{L}^{-1}$, tal inclusão, deve-se a uma preocupação com os possíveis efeitos danosos a saúde dos seres humanos.

A importância da realização de estudos sobre os efeitos tóxicos de compostos químicos, utilizando organismos sensíveis, compostos estes passíveis de virem a ser microcontaminantes emergentes de águas superficiais e potáveis, os quais estão relacionados com a concentração e suas propriedades, bem como estes compostos podem afetar o organismo exposto, esta centrada no estabelecimento do potencial tóxico, no mecanismo de ação nos seres vivos e nas taxas de sensibilidade para diferentes organismos aquáticos, em relação à mesma substância. Com este enfoque, a pesquisa objetiva avaliar os efeitos ecotoxicológicos de um herbicida comercial composto pelos principio ativos diuron $\mathrm{e}$ hexazinona, atualmente empregados na cultura da cana-de-açúcar, utilizando como organismo testes neonatas de Ceriodapnhia dubia para determinar valores da concentração efetiva média $\left(\mathrm{CE}_{50}\right)$ desses compostos.

\section{MATERIAIS E MÉTODOS}

A metodologia empregada na realização dos ensaios de toxicidade aguda em Ceriodaphnia dubia foi baseada nas NBR 12.713 (ABNT 2004) e NBR 13.373 (ABNT 2005), utilizando como 
contaminantes um herbicida comercial e seus dois compostos, Diuron e Hexazinona isoladamente.

\subsection{PREPARO DA ÁGUA DE DILUIÇÃO}

Uma água reconstituída foi utilizada nos ensaios para diluições de contaminantes e para o cultivo dos organismos testes, esta água foi preparada em laboratório com adição de reagentes químicos para obtenção de valores de $\mathrm{pH}$ de 7,3 \pm 0,3 e dureza de $44 \pm 4 \mathrm{mg} \mathrm{CaCO}_{3} \cdot \mathrm{L}^{-1}$ e mantida em temperatura de $25{ }^{\circ} \mathrm{C} \pm 2$, para o controle destes valores, estes parâmetros foram analiticamente verificados semanalmente (APHA, 2005). Na Tabela 1 estão apresentadas as quantidades dos reagentes químicos de pureza analítica que foram utilizados no preparo da água reconstituída do cultivo.

Após preparação da água reconstituída a mesma foi aerada por $12 \mathrm{~h}$ através de um compressor de ar, para proporcionar solubilização dos sais, saturação do oxigênio dissolvido e estabilização do pH. Dessa água reconstituída, foram mantidas soluções-estoque aeradas constantemente em frasco de $1000 \mathrm{~mL}$, para posteriormente serem utilizadas no cultivo dos organismos-testes que necessita de uma renovação da água três vezes por semana, com uma troca total e outras duas parciais de $50 \%$, tendo-se o cuidado no manuseio para evitar alterações na temperatura da água $\left( \pm 2^{\circ} \mathrm{C}\right)$ para não causar danos aos organismos-testes.

\subsection{PREPARAÇÃO DOS ALIMENTOS PARA OS ORGÂNISMOS}

De acordo com a metodologia aplicada, o alimento utilizado para os organismos-testes foram algas verdes Pseudokirchneriella subcapitata e um alimento composto à base de ração comercial para peixe e fermento biológico. $O$ fornecimento do alimento no cultivo das algas foi diário, com dosagens controladas, evitando que os organismos permanecessem mais de $48 \mathrm{~h}$ sem alimentação e/ou com excesso de alimentos.

Para o preparo do meio de cultivo das algas foram adicionados $30 \mathrm{~mL}$ de meio $\mathrm{CHU}$ (CHU, 1942) em 1,5 L de água destilada, cujas soluções de preparo foram esterilizadas em autoclave na temperatura de $120^{\circ} \mathrm{C}$ e pressão de $1 \mathrm{~atm}$, por 20 minutos. Após resfriamento dessas soluções, foram adicionadas iscas de culturas da alga para a sua posterior reprodução. Na Figura 1, é apresentado o frasco com o cultivo das algas.

Para a obtenção de células viáveis, as culturas de algas Pseudokirchneriella subcapitata foram mantidas em temperatura entre $20^{\circ}$ a $25^{\circ} \mathrm{C}$, com permanente aeração e iluminação artificial com 4 lâmpadas fluorescentes compactas de $25 \mathrm{~W}$, após atingirem o crescimento adequado, em aproximadamente 7 dias, a cultura foi centrifugada e o sobrenadante do meio de cultura das algas foi descartado, as algas sedimentadas foram coletadas e posteriormente levadas à câmara de Neubauer para contagem do número de células viáveis por $\mathrm{mL}$.
TABELA 1: Quantidade de reagentes químicos de pureza analítica (PA) utilizados na preparação da água reconstituída com diluição para 1000 mL no cultivo das Ceriodaphnia dubia.

\begin{tabular}{lcc} 
Reagente P.A. & Fórmula molecular & $\begin{array}{c}\text { Quantidade } \\
(\mathrm{mg})\end{array}$ \\
\hline Sulfato de cálcio & $\mathrm{CaSO}_{4} \cdot 2 \mathrm{H}_{2} \mathrm{O}$ & 1500 \\
Cloreto de potássio & $\mathrm{KCl}$ & 200 \\
Bicarbonato de sódio & $\mathrm{NaHCO}_{3}$ & 4800 \\
Sulfato de magnésio & $\mathrm{MgSO}_{4} .7 \mathrm{H}_{2} \mathrm{O}$ & 6100 \\
\hline
\end{tabular}

Fonte: NBR 13.373 (ABNT, 2005).

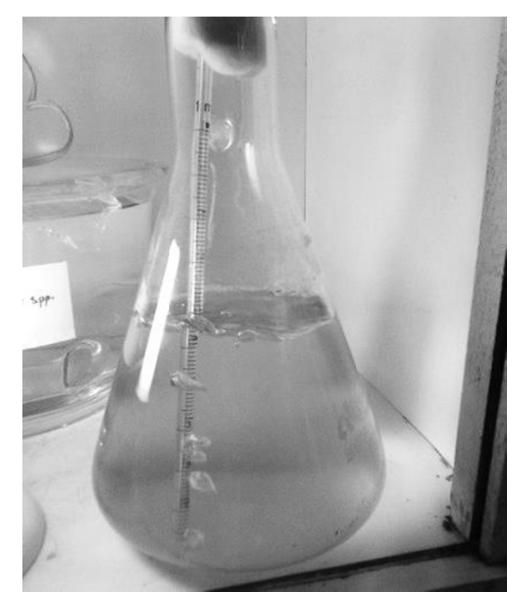

FIGURA 1: Manutenção de meio de cultura de algas Pseudokirchneriella subcapitata. 
No fornecimento do alimento aos organismos teste, à base de algas verdes, utilizou-se uma dosagem de 1 a $5 \times 10^{5}$ células por organismo, diariamente, de acordo com as recomendações da NBR 13.373 (ABNT, 2005).

Com o objetivo de otimizar o processo de contagem das algas ( $\mathrm{n}$ - de células. $\mathrm{mL}^{-1}$ ), foi desenvolvida uma técnica analítica alternativa por espectrofotometria na região de UV-visível em um equipamento Espectrofotômetro Cary $1 \mathrm{E}$, da marca Varian, com cubeta de quartzo de $1 \mathrm{~cm}$ de caminho óptico. Uma varredura da cultura na região de UVvisível foi empregada para a determinação do comprimento de onda de absorção máxima, em seguida, foi feita uma curva de calibração com diferentes concentrações conhecidas de alga obtendo-se uma correlação entre absorbância e número de células por $\mathrm{mL}$ (PASCHOALATO et al. 2013).

No preparo do alimento composto, utilizou-se $5 \mathrm{~g}$ de ração comercial para peixe dissolvida em $1 \mathrm{~L}$ de água destilada, mantido sob aeração por 7 dias, o qual foi filtrado em papel qualitativo e posteriormente separado em porções de $50 \mathrm{~mL}$, armazenados em frascos plásticos descartáveis e mantidos em temperatura de -4 - $\mathrm{C}$ (em freezer). Para a utilização, esperou-se atingir a temperatura ambiente natural e foi adicionado
0,25 g de fermento biológico mais $50 \mathrm{~mL}$ de água destilada para formar $100 \mathrm{~mL}$. Esse composto, em seguida, foi novamente filtrado em papel qualitativo e armazenado em geladeira sendo consumido em até uma semana.

\subsection{MANUTENÇÃO DE ORGANISMOS TESTES E ENSAIOS DE TOXICIDADE AGUDA}

Os organismos foram mantidos em laboratório climatizado $\left(23^{\circ} \mathrm{C}\right)$ em recipientes de vidro e divididos em lotes de até 70 adultos por litro, os recipientes foram cobertos por filme plástico com furos. Foi utilizado um equipamento de foto período para controle de $16 \mathrm{~h}$ de luz em todas as culturas de Ceriodaphnias dubias. A Figura 2 mostra os recipientes utilizados para a manutenção dos organismos-testes. Desses recipientes obtiveram-se as neonatas de Ceriodaphnias dubia que foram utilizadas nos testes agudos.

A manutenção e a separação das neonatas de Ceriodaphnias dubia objetivou um melhor desempenho na reprodução, a fim de evitar o excesso de população $e$ „posteriormente, serem utilizadas para avaliar a toxicidade aguda de substâncias químicas em contato com esses organismos. Na Figura 3 podemos ver um exemplar de Ceriodaphnia dubia (MBL, 2005).

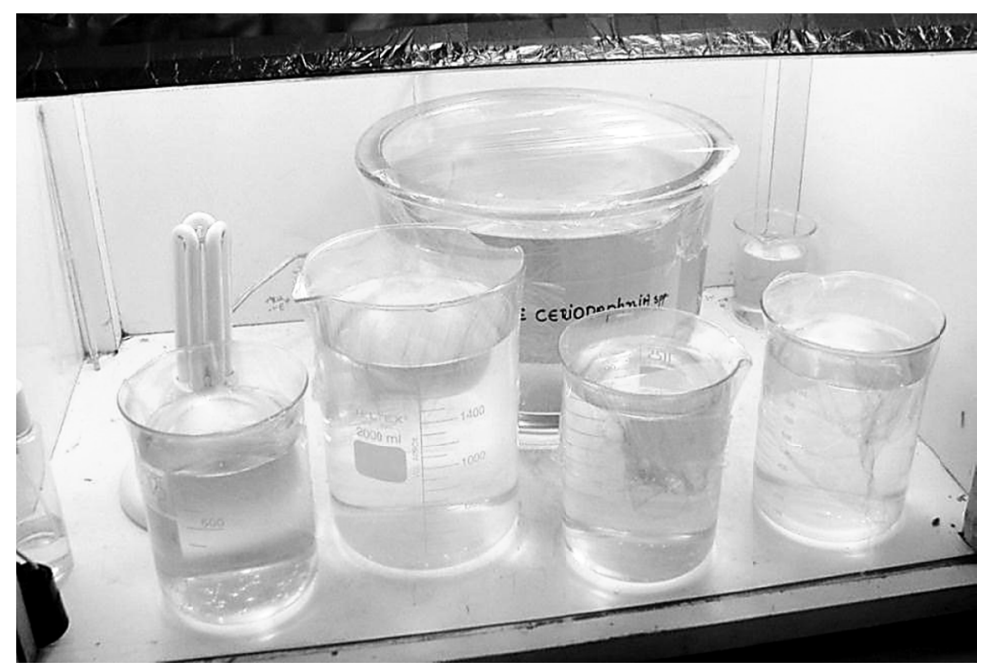

FIGURA 2: Manutenção dos organismos-testes.

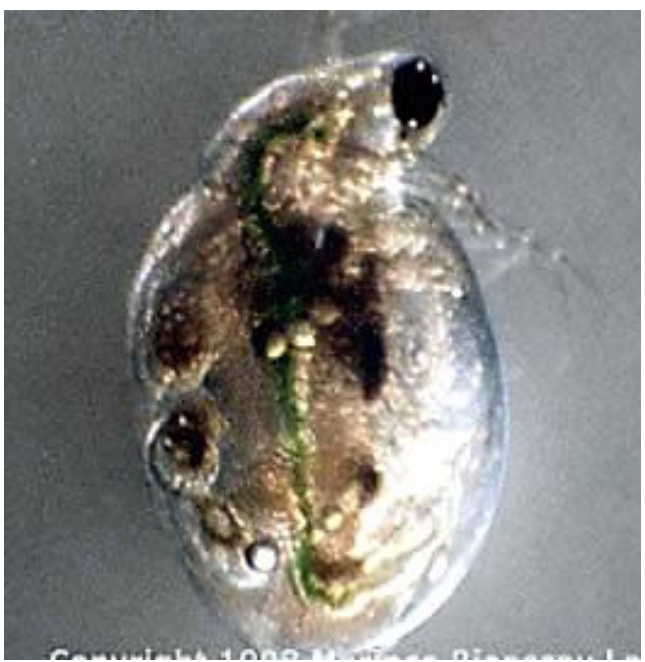

FIGURA 3: Exemplar de Ceriodaphnia dubia. Fonte: MBL Aquaculture (2005). 
A separação dos organismos de Ceriodaphnias dubia ocorreu a cada 3 dias, considerando a idade e diferentes lotes. O procedimento de separação das Ceriodaphnias dubias adultas das neonatas (de 6 a $24 \mathrm{~h}$ de vida), foi realizado com 0 auxílio de uma pipeta automática com ponteira descartável, onde as neonatas eram capturadas e inseridas em uma nova solução de água reconstituída, a fim de serem posteriormente utilizadas nos testes agudos. A justificativa da utilização apenas de organismos neonatas nos testes agudos é por serem mais sensíveis aos agentes químicos do que os organismos adultos, e por haver diferenças no grau de desenvolvimento dos mecanismos de desintoxicação entre organismos jovens e adultos (FORGET et al., 2005).

Os ensaios de toxicidade aguda avaliam uma resposta severa e rápida dos organismos aquáticos a um estímulo que se manifesta, em geral, num intervalo de 0 a $96 \mathrm{~h}$ (RAND et al., 1995). Neste trabalho, o tempo de exposição estudado foi de 48 h (CETESB, 1991; USEPA, 2001 e ABNT, 2004).

Para os ensaios de toxicidade aguda foram selecionados organismos neonatas de Ceriodaphnia dubia, de 6 a $24 \mathrm{~h}$ de idade, e estes foram expostos ao contaminante por um período de $48 \mathrm{~h}$, sem iluminação e sem o fornecimento de alimento. Para a separação dos organismos neonatas, com idade desejada, novamente foi necessária à aspiração com auxilio de um pipetador, um dia antes de iniciar os testes agudos, de um lote de fêmeas ovadas, posteriormente transferidas para frascos que foram mantidos a $23^{\circ} \mathrm{C}$, sendo alimentadas com algas Pseudokirchneriella subcapitata em uma concentração de aproximadamente $2 \times 10^{5}$ e de alimento composto com $2 \mathrm{~mL} . \mathrm{L}^{-1}$.

Os efeitos observados nos organismos testes são a imobilização ou a letalidade. É importante ressaltar que são considerados imóveis, além dos organismos aparentemente mortos, aqueles incapazes de nadar na coluna d'água em até 15 segundos após leve agitação do recipiente. Para cada teste definitivo de toxicidade aguda, foi observada a imobilização dos organismos para determinação da $\mathrm{CE}_{50}-48 \mathrm{~h}$ (concentração efetiva média), isto é, a concentração do agente tóxico que causa imobilidade estatisticamente a $50 \%$ dos organismos teste depois de 48 horas de exposição ao contaminante.

Os testes de toxicidade aguda para o herbicida comercial foram realizados partindo-se de uma solução de herbicida comercial preparada para a obtenção de uma concentração de $25 \mathrm{mg} \cdot \mathrm{L}^{-1}$, adotado em função da composto com menor solubilidade (diuron $42 \mathrm{mg} / \mathrm{L}$ ), em seguida foram preparadas, por diluições, 26 soluções de concentrações que variaram de 0,50 a $13 \mathrm{mg} \cdot \mathrm{L}^{-1}$, sendo essas concentrações baseadas em ensaios preliminares.

Nos ensaios de toxicidade aguda para diuron, partiu-se de uma solução de $25 \mathrm{mg} \cdot \mathrm{L}^{-1}$, em seguida foram diluídas para obtenção de 15 concentrações variando entre 0,25 a $12 \mathrm{mg} \cdot \mathrm{L}^{-1}$ e nos ensaios de toxicidade aguda para hexazinona, partiu-se de uma solução de $1.000 \mathrm{mg} \cdot \mathrm{L}^{-1}$, que por diluições obteve-se 15 diferentes concentrações variando entre 1 a $500 \mathrm{mg} . \mathrm{L}^{-1}$. Em todos os ensaios foi inserido um controle (sem contaminação) e os mesmos foram realizados em triplicata. Os recipientes utilizados foram frascos de vidro com capacidade para $25 \mathrm{~mL}$, conforme a Figura 4 .

Foram colocadas nesses frascos $20 \mathrm{~mL}$ das soluções diluídas dos herbicidas comercial, puro e do controle, em seguida, foram transferidos 5 organismos neonatos para cada frasco com o auxílio de uma pipeta automática com ponteira descartável e acondicionados em estufa incubadora com temperatura controlada entre $23^{\circ} \mathrm{C}$ a $25^{\circ} \mathrm{C}$.

Durante o período de testes, os frascos foram mantidos sem alimentação e ausência total de iluminação, sendo que as variáveis físicas e químicas ( $\mathrm{pH}$, dureza total e oxigênio dissolvido) foram controladas semanalmente durante 0 decorrer dos testes.

Após 48 horas, com o auxilio de uma lupa, observou-se e quantificou-se o número de indivíduos imóveis por frascos, em triplicata. 0 método estatístico Trimmed Spearman-Karber Method for Estimating Median Lethal Concentrations in Toxicity Biossays (HAMILTON et al., 1977; APHA, 2005), foi utilizado para cálculo dos 
valores de $\mathrm{CE}_{50}$, que são relativos à imobilização dos organismos testes, que correspondem às concentrações das amostras no início do ensaio com efeito de toxicidade aguda a $50 \%$ dos organismos expostos a $48 \mathrm{~h}$.

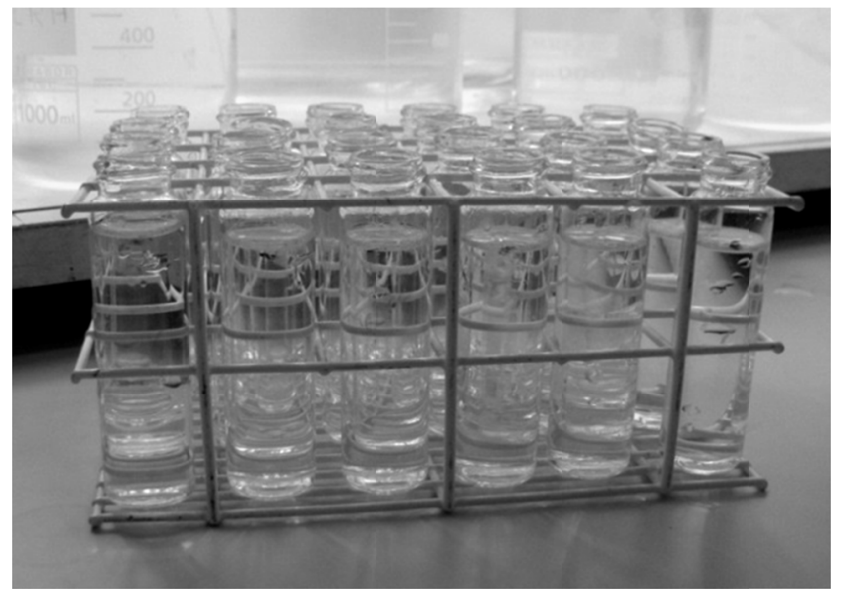

FIGURA 4: Frascos de vidro utilizados nos testes agudos.

Os métodos Spearman-Karber e Trimmed Spearman-Karber são métodos não paramétricos que têm boas propriedades estatísticas, são fáceis de usar e são recomendados para cálculos precisos de $\mathrm{CE}_{50}$ (Concentração Efetiva Média) e $\mathrm{CL}_{50}$ (Concentração Letal Média) com intervalo de confiança de 95\% (HAMILTON et al., 1977). Os valores numéricos de toxicidade aguda, expressos em $\mathrm{CE}_{50}$ exprimem uma relação inversa com a toxicidade, ou seja, quanto menor esse valor maior a toxicidade (SILVA et al., 2012).

O método estatístico de Trimmed Spearman-Karber é válido para curvas dose-resposta simétricas e assimétricas. A única limitação desse método em relação aos métodos paramétricos é que deve cobrir o intervalo de zero a $100 \%$ de mortalidade ou de efeito agudo (HAMILTON et al., 1977; APHA, 2005; BAEZ et al., 2004).

\subsection{ENSAIOS DE SENSIBILIDADE DOS ORGANISMOS TESTES}

De acordo com Santos et al. (2007), estudos mostraram que cloreto de sódio $(\mathrm{NaCl})$ pode ser usado como um produto tóxico de referência, atendendo à dois requisitos importantes em uma pesquisa: o $\mathrm{NaCl}$ é menos perigoso para a manipulação humana e para o ambiente quando comparado ao tradicional dicromato de potássio e a outros, e sua reprodutibilidade é aceitável em testes laboratoriais de rotina.

Os organismos testes foram avaliados em relação à sensibilidade a fim de assegurar a qualificação dos mesmos dentro dos padrões internacionais e garantir a validação dos testes realizados exigidos pela norma da ABNT.

No ensaio de sensibilidade, a substância empregada foi o cloreto de sódio de dureza analítica ( $\mathrm{NaCl} \mathrm{PA})$, foram realizados 5 ensaios, em duplicata, com 5 concentrações e um controle, variando entre 0,6 a 2,2 g. $\mathrm{L}^{-1}$ de $\mathrm{NaCl}$, os valores obtidos foram utilizados no cálculo do $\mathrm{CE}_{50}$ para o $\mathrm{NaCl}$. Os resultados devem compreender um intervalo de \pm 2 desvios-padrão em relação aos valores médios anteriormente obtidos para a mesma espécie.

\section{RESULTADOS E DISCUSSÃO}

Por meio da realização de um espectro (varredura) entre os comprimentos de onda de 200 a $800 \mathrm{~nm}$, determinou-se o valor do comprimento de onda de máxima absorção de $685 \mathrm{~nm}$, considerando a coloração esverdeada da solução. Este comprimento de onda foi utilizado na construção da curva de calibração pelo método espectrofotométrico.

Partindo-se de uma concentração de algas de $2,05 \times 10^{7} \mathrm{n}$ o células. $\mathrm{mL}^{-1}$, previamente determinada na câmara de Neubauer, foram feitas diluições de concentrações decrescentes para a construção da curva de calibração de absorbância em função do $\mathrm{n}$ - células por $\mathrm{mL}$.

O coeficiente de correlação $\left(R^{2}\right)$ obtido foi de 0,9995 , indicando que a técnica analítica apresentou uma linearidade satisfatória, conforme mostrado na Figura 5, permitindo a quantificação do número de células por $\mathrm{mL}$ de maneira rápida e com confiabilidade. De acordo com ANVISA, (2003) o critério mínimo aceitável do coeficiente de correlação deve ser de superior ou igual a 0,99. 


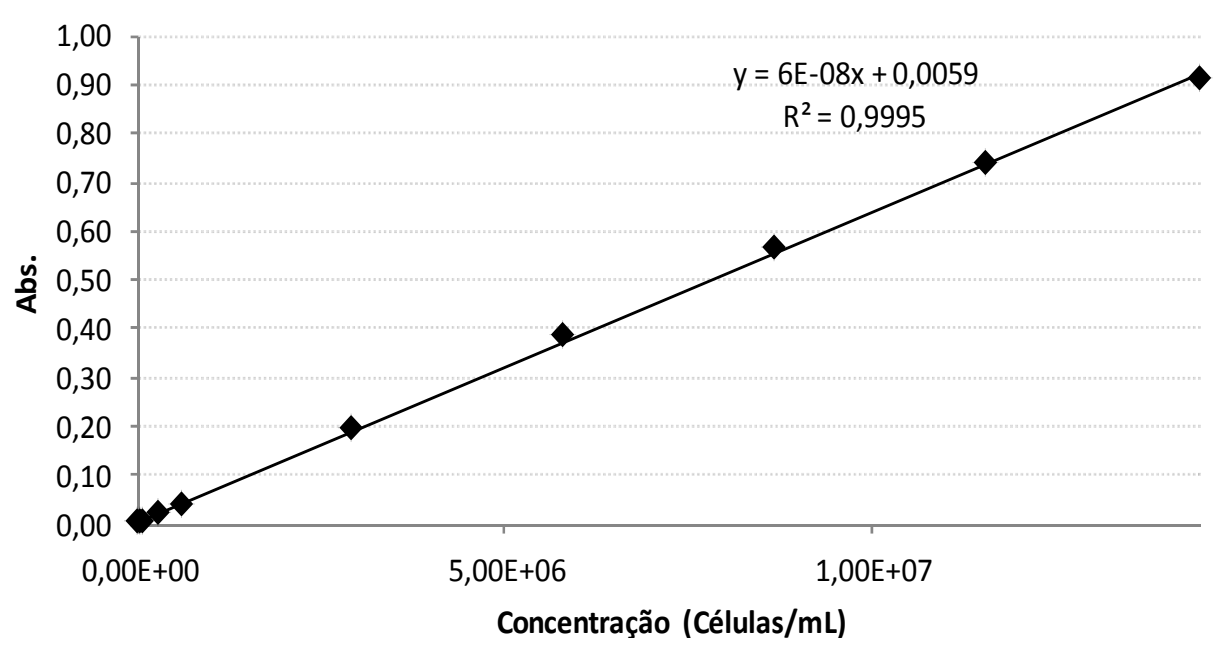

FIGURA 5: Curva de calibração do método alternativo de contagem de algas para a alimentação dos organismos-testes. Fonte: Paschoalato et al. 2013.

Nos testes agudos com neonatas de Ceriodaphnia dubia, primeiramente, foi avaliado como contaminante o herbicida comercial, utilizando-se 26 concentrações e mais uma solução para controle. Posteriormente, os compostos ativos do herbicida comercial foram avaliados separadamente, sendo o Diuron e o Hexazinona, ambos em 15 concentrações distintas e mais uma solução de controle para cada composto. Em todos os testes foram observados os $\mathrm{CE}_{50}-48 \mathrm{~h}$, a partir das porcentagens de imobilização dos organismos testes expostos por $48 \mathrm{~h}$ a várias concentrações destes contaminantes.

$\mathrm{O}$ valor obtido de $\mathrm{CE}_{50}-48 \mathrm{~h}$ para os testes agudos com um herbicida comercial foi de 5,78 mg. $L^{-1}$, o qual representa estatisticamente $50 \%$ de imobilização dos organismos testes. A Figura 6 demonstra a concentração do herbicida comercial em função da porcentagem de imobilidade dos organismos testes, a faixa de toxicidade variou de $1,5 \mathrm{mg} \cdot \mathrm{L}^{-1}$, que representa a concentração máxima que não causa imobilidade aos organismos a 11,5 mg. $\mathrm{L}^{-1}$, a qual representa a concentração que causa $100 \%$ de imobilidade aos organismos.
De acordo com Silva et al. (2010), em um trabalho no qual foi utilizado o mesmo herbicida comercial e os organismos teste foram peixes Danio rerio, obteve-se como resposta do $\mathrm{CL}_{50}$ um valor de $581,50 \mathrm{mg} . \mathrm{L}^{-1}$. O mesmo trabalho também detectou que a partir de $50 \mathrm{mg} \cdot \mathrm{L}^{-1}$ desse herbicida, houve alterações comportamentais significativas, como hipoatividade, relacionada ao movimento natatório em mais de $50 \%$ dos organismos. Portanto os resultados encontrados com o mesmo herbicida comercial demonstraram que as espécies Ceriodapnhias dubia, sendo de menor tamanho corpóreo e de nível trófico anterior, sofreram maiores efeitos tóxicos do que comparado as espécie de peixes Danio rerio. Segundo Williamson (1980), os animais pequenos apresentam uma maior taxa metabólica.

Os resultados dos testes com os componentes puros Diuron e Hexazinona, foram realizados separadamente, com propósito de uma avaliação específica de seus efeitos tóxicos. $\mathrm{Na}$ Figura 7 estão apresentados os resultados obtidos com o Composto Diuron e o valor obtido de $\mathrm{CE}_{50}$ que foi de aproximadamente $2,9 \mathrm{mg} \cdot \mathrm{L}^{-1}$. 


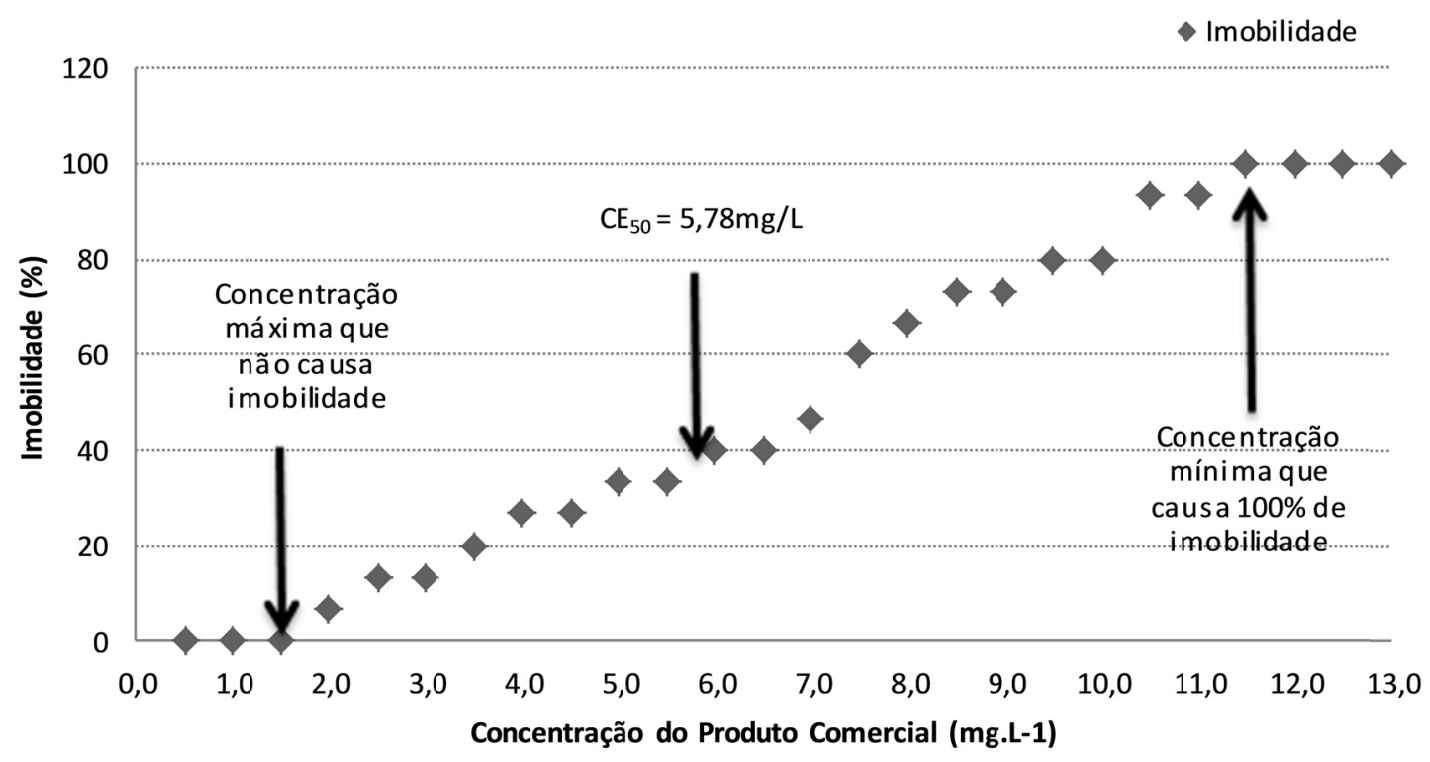

FIGURA 6: Concentração do herbicida comercial em função da porcentagem de imobilidade dos organismos testes.

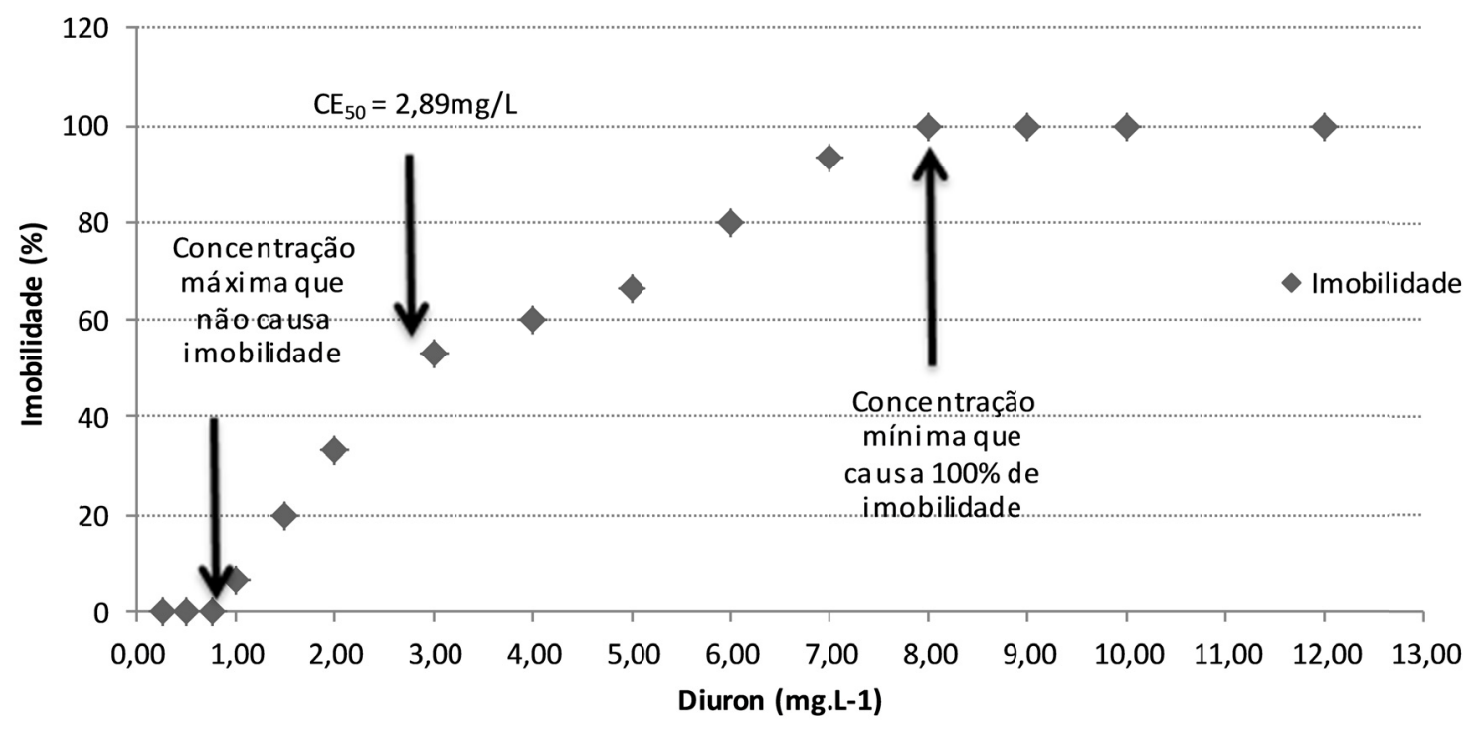

FIGURA 7: Resultado do valor de $\mathrm{CE}_{50}$ nos testes agudos com o herbicida diuron com porcentagem de imobilidade dos organismos testes em função da concentração.

Na Figura 8 estão apresentados os resultados obtidos nos testes agudos com Ceriodapnhia dubia expostas em hexazinona e o valor obtido de $\mathrm{CE}_{50}$ foi de aproximadamente 105,56 mg. $\mathrm{L}^{-1}$.

De acordo com dados da USEPA (1997 e 2003) foram encontrados valores de $C_{50}$ para diuron de $1,4 \mathrm{mg} . \mathrm{L}^{-1}$ e para hexazinona valores de $\mathrm{CL}_{50}$ de 151,6 mg. $\mathrm{L}^{-1}$ em testes agudos com Dapnhia magna, esses valores comparados aos obtidos no trabalho demonstram variações no valor de $\mathrm{CL}_{50}$. Segundo dados na literatura, as condições do meio, por serem espécies de diferentes sensibilidades a um mesmo contaminante ou a contaminantes diferentes, valores de $\mathrm{CL}_{500}$ podem ser passíveis de variações, não podendo assim ser justificados baseando-se em apenas um dado.

Nas Tabelas 2 e 3, estão apresentadas as compilações de valores de $\mathrm{CE}_{50}$ e $\mathrm{CL}_{50}$ para diferentes organismos testes, expostos aos 
herbicidas diuron e hexazinona respectivamente, em trabalhos realizados por diferentes autores e classificados por ordem trófica.

Dentre os valores de $\mathrm{CE}_{50}$ e $\mathrm{CL}_{50}$ encontrados na literatura, a faixa de toxicidade do diuron para algas variou entre 0,0007 a 0,070 mg. L' 1; para os cladoceras essa faixa ficou em 2,9 a $21,117 \mathrm{mg} / \mathrm{L}$ e, para os peixes, variou de 4,3 a 42 $\mathrm{mg} . \mathrm{L}^{-1}$ respectivamente. As espécies de algas foram mais afetadas pelo diuron, por ser um herbicida, o diuron tem seu efeito direcionado para a inibição da fotossíntese, consequentemente prejudicando o crescimento de algas. Os invertebrados aquáticos, por serem de níveis tróficos anteriores as Dapnhias, apresentaram níveis maiores de sensibilidade à toxicidade do diuron.

Os organismos aquáticos que apresentaram maior sensibilidade ao diuron foram as Ceriodapnhias dubia, dentre as cladoceras, pois são espécies com menor tamanho corpóreo, filtradores na cadeia alimentar, apresentando assim, uma maior sensibilidade à exposição, do que espécies de tamanho corpóreo maior (WILLIAMSON, 1980; FREITAS e ROCHA, 2012). Além disso, a reprodução assexuada desses crustáceos por partenogênese garante a produção de organismos geneticamente idênticos, permitindo assim a obtenção de organismos-teste com sensibilidade constante (TATARAZAKO et al., 2003; BURATINI et al., 20014).

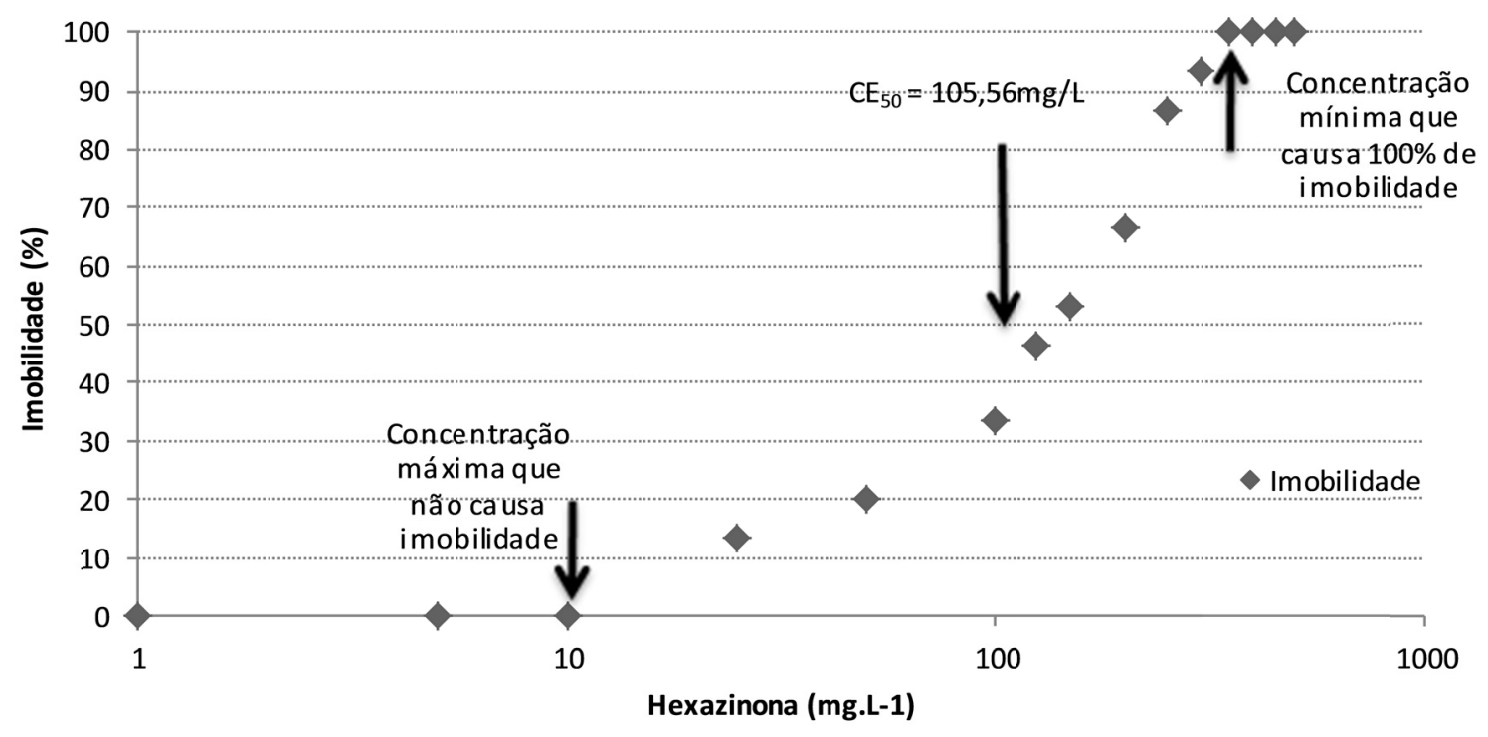

FIGURA 8: Resultados do valore de $\mathrm{CE}_{50}$ nos testes agudos com hexazinona com a porcentagem de imobilidade em função da concentração.

TABELA 2: Referência de valores de CE50 e CL50 para diferentes organismos, organizados por níveis tróficos, expostos ao diuron.

\begin{tabular}{|c|c|c|c|}
\hline \multirow{2}{*}{ Organismos testes } & \multicolumn{2}{|c|}{ Diuron } & \multirow{2}{*}{ Referências } \\
\hline & $\mathrm{CE}_{50}(\mathrm{mg} / \mathrm{L})$ & $\mathrm{CL}_{50}(\mathrm{mg} / \mathrm{L})$ & \\
\hline Invertebrados aquáticos & & 1 a $2,5-48 h$ & Giacomazzi, 2004 \\
\hline Scenedesmus vacuolatus & 0,05 a $0,07-24 h$ & & Neuwoehner et al., 2008 \\
\hline Selenastrum capricornotum & $0,045-72 h$ & & Fernandez-Alba, 2002 \\
\hline Raphidocelis subcapitata & $0,0007-96 h$ & & Ma, et al., 2006 \\
\hline Cladoceras em geral & & $21,117-48 h$ & Sanchez-Bayo, 2006 \\
\hline Ceriodapnhia dubia & $2,9-48 h$ & & Presiente estudo \\
\hline Dapnhia magna & $8,6-48 h$ & & Fernandez-Alba, 2002 \\
\hline Dapnhia pulex & & $17,9-96 h$ & Nebieker, 1998 \\
\hline Hyalella azteca & & $19,4-96 h$ & Nebieker, 1998 \\
\hline Truta arco-íris & & $3,5-96 h$ & Giacomazzi, 2004 \\
\hline Peixes & & 4,3 a $42-48 h$ & Giacomazzi, 2004 \\
\hline
\end{tabular}


XTABELA 3: Valores de CE50 e CL50 para diferentes organismos, organizados por níveis tróficos, expostos

\section{a hexazinona.}

\begin{tabular}{|c|c|c|c|}
\hline \multirow{2}{*}{ Organismos testes } & \multicolumn{2}{|c|}{ Hexazinona } & \multirow{2}{*}{ Referências } \\
\hline & $\mathrm{CE}_{50}(\mathrm{mg} / \mathrm{L})$ & $\mathrm{CL}_{50}(\mathrm{mg} / \mathrm{L})$ & \\
\hline Selenastrum capricornutum & $0,126-168 h$ & & Montague, 2000 \\
\hline Cladoceras em geral & & $130,88-48 h$ & Sanchez-Bayo, 2006 \\
\hline Ceriodapnhia dubia & $105,56-48 h$ & & Presiente estudo \\
\hline Dapnhia magna & $151,6-48 h$ & & Montague, 2000 \\
\hline Oncorhynchus gorbuscha & & $839-48 h$ & Wan et al., 1988 \\
\hline Oncorhynchus gorbuscha & & $728-72 h$ & Wan et al., 1988 \\
\hline Oncorhynchus keta & & $286-48 h$ & Wan et al., 1988 \\
\hline Oncorhynchus keta & & $146,7-96 h$ & Montague, 2000 \\
\hline
\end{tabular}

Os valores de $\mathrm{CE}_{50}$ e $\mathrm{CL}_{50}$ para Hexazinona testados em organismos das espécies cladocera, ficaram na faixa de 105,56 a $151,6 \mathrm{mg} \cdot \mathrm{L}^{-1}$, os demais organismos, apresentaram valores entre 146,7 a $839 \mathrm{mg} . \mathrm{L}^{-1}$, sendo assim mais resistentes. Portanto, os organismos de níveis tróficos acima dos cladoceras, na cadeia alimentar, apresentam maior resistência aos efeitos tóxicos à Hexazinona.

As algas são drasticamente afetadas pelos efeitos tóxicos dos herbicidas diuron e hexazinona que são inibidores da fotossíntese. As Ceriodapnhia dubia são animais de tamanho corpóreo pequeno e com alta taxa metabólica, filtradores do zooplâncton e consumidores primários na cadeia alimentar, mostrando neste trabalho serem organismos de alta sensibilidade à alteração do meio e a presença dos herbicidas estudados.

Foram estabelecidos valores de concentrações, através dos testes de sensibilidade para os organismos utilizando o cloreto de sódio $(\mathrm{NaCl})$. A partir desses resultados adquiridos de cada 5 concentrações testadas, foram calculados os $\mathrm{CE}_{50} 48 \mathrm{~h}$ destas concentrações nos testes de sensibilidade.

Na Figura 9 está demonstrada a faixa de sensibilidade obtida para a Ceriodaphnia dubia sob exposição ao $\mathrm{NaCl}$. A linha central representa o valor médio das $\mathrm{CE}_{50}-48 \mathrm{~h}$ obtidas de $1,50 \mathrm{~g} \cdot \mathrm{L}^{-1}$ de $\mathrm{NaCl}$, as linhas tracejadas inferior e superior demonstram os intervalos de sensibilidade que variou entre 1,33 e 1,68 g. $\mathrm{L}^{-1}$ de $\mathrm{NaCl}$, obtendo-se os desvios-padrões aceitáveis exigidos pela ABNT.

Todos os valores se apresentaram dentro do desvio padrão esperado, sendo assim os testes de sensibilidade asseguraram a qualidade dos organismos-testes e a confiabilidade dos resultados.

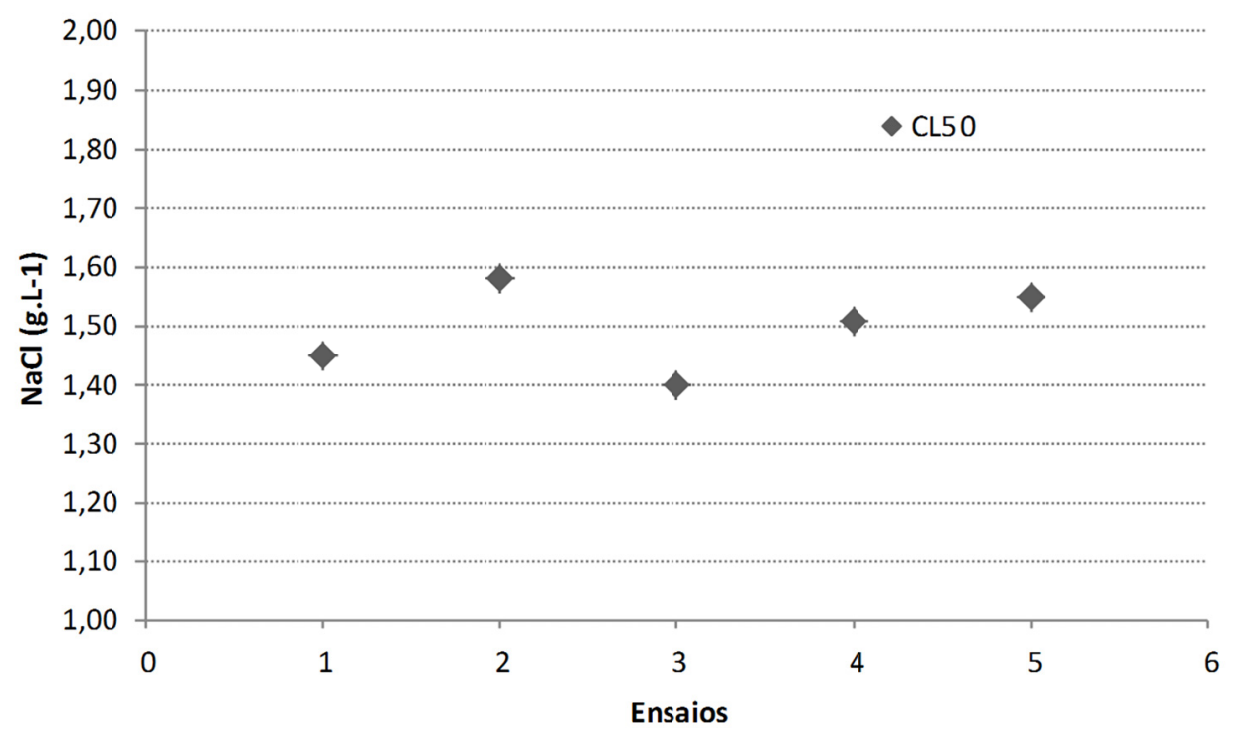

FIGURA 9: Resultados das $\mathrm{CE}_{50}-48 \mathrm{~h}$ dos testes de sensibilidade da Ceriodaphnia dubia a exposição com o $\mathrm{NaCl}$. 


\section{CONSIDERAÇÕES FINAIS}

Pela importância econômica da cultura da cana-de-açúcar para o desenvolvimento do país combinada a preocupação em preservação dos recursos hídricos visando os usos múltiplos $\mathrm{e}$ sustentabilidade, pelos resultados dos testes de toxicidade para o herbicida comercial e dos compostos diuron e hexazinona, pode-se concluir que o organismo teste utilizado, Ceriodaphnia dubia, apresentou uma facilidade de cultivo em laboratório, sensibilidade durante as realizações dos testes e reprodutividade, mostrando a viabilidade de sua utilização em testes de ecotoxicidade.

Os testes de sensibilidade apresentaram-se dentro do intervalo de confiabilidade exigido pela norma da ABNT NBR 12713, concluindo-se pela validação dos resultados encontrados para os compostos estudados. Os testes agudos mostraram que os herbicidas em estudo, são microcontaminantes e apresentam toxicidade para organismos aquáticos no nível trófico primário da cadeia alimentar.

Nos resultados obtidos para $\mathrm{CE}_{50}-48 \mathrm{~h}$ em testes com Ceriodaphnia dubia foram obtidos valores para diuron de $2,9 \mathrm{mg} \cdot \mathrm{L}^{-1}$ e para hexazinona de $105,56 \mathrm{mg} \cdot \mathrm{L}^{-1}$, conclui-se que diuron tem potencial tóxico, aproximadamente 37 vezes superior, quando comparado a hexazinona.

$\mathrm{O}$ produto herbicida comercial teve o seu resultado de $\mathrm{CE}_{50}-48 \mathrm{~h}$ de $5,78 \mathrm{mg} \cdot \mathrm{L}^{-1}$, podendo-se concluir que a presença de $13,2 \%$ de hexazinona na mistura tenha proporcionado uma redução da toxicidade em relação ao diuron puro nos organismos testes.

Avaliando-se os valores obtidos de toxicidade por diversos trabalhos consultados na literatura, mesmo considerando organismos pertencentes a diferentes cadeias tróficas, os valores de $\mathrm{CE}_{50}$ apresentaram-se próximos, porem, faz se necessário à compilação de diversos ensaios de diferentes níveis tróficos, no intuito de gerar dados para as avaliações de análise de risco, afim de que os órgãos regulamentadores possam, com segurança, estabelecer limites máximos permissíveis para qualidade das águas superficiais na legislação brasileira.

\section{REFERÊNCIAS BIBLIOGRÁFICAS}

ABNT - ASSOCIAÇÃO BRASILEIRA DE NORMAS TÉCNICAS. Norma NBR 12713: Ecotoxicologia aquática Toxicidade aguda - Método de ensaio com Daphnia spp (Cladocera, Crustacea). 21p. Rio de janeiro-RJ, 2004.

ABNT - ASSOCIAÇÃO BRASILEIRA DE NORMAS TÉCNICAS. Norma NBR 13373: Ecotoxicologia Aquatica - Toxicidade crônica - Método de Ensaio com Ceriodaphnia spp. (Cladocera, Crustácea). 15 p. Rio de Janeiro, 2005.

APHA, AWWA, WPCF - American Public Health Association; American Water Works Association; Water Publuic Environment Federation. Standard Methods for the Examination of Water and Wastewater. $21^{\text {th }}$ Washington, D.C., 2005.

ANVISA - Agência Nacional de Vigilância Sanitária; Resolução $n^{\circ}$ 899, de 29 de maio de 2003: Guia para validação de métodos analíticos e bioanalíticos, Ministério da Saúde: Brasil, 2003.

ARMAS, E.D. Biogeodinâmica de herbicidas utilizados em cana-de-açúcar (Saccharumssp.) na sub-bacia do rio Corumbataí. (Tese de doutorado). Escola Superior de Agricultura Luiz de Queiroz, 186 p., Piracicaba, SP. 2006.

BAEZ, M.C.D.; ROSSINI, G.D.B. \& GRANADOS, Y.P. 2004. Ensayos Toxicológicos y Métodos de Evaluacion de Calidad de Aguas - Estandarizacion, Intercalibracion, Resultados y Aplicaciones. Centro Internacional de Investigaciones para el Desarrollo, Ottawa, Canada.

BRASIL. Conselho Nacional do Meio Ambiente no 357, de 31 de março de 2005. Classificação dos corpos de água e diretrizes ambientais para o seu enquadramento, bem como estabelece as condições e padrões de lançamento de efluentes, e dá outras providências. Brasília: Diário Oficial da República Federativa do Brasil, 2005.

BRASIL, Ministério da Saúde, Secretaria de Vigilância em Saúde, Portaria MS n. ${ }^{\circ} 2914$, de 12 de dezembro de 2011. Dispõe sobre os procedimentos de controle e de vigilância da qualidade da água para consumo humano e seu padrão de potabilidade. Diário Oficial da União, Brasília, DF, 2011.

BURATINI, S.V.; BERTOLETTI, E.; ZAGATTO, P.A. 2004. Evaluation of Dapnhia similis as a test species in ecotoxicological assays. Bull. Environ. Contam. Toxicol., 73(5): 878-882. 
CHU, S. P. 1942. The influence of the miceral composition of the medium on the growth of planktonic algae. L-methods and culture media. Journal of Ecology, n 30, p.284-325.

CETESB- Companhia de Tecnologia e Saneamento Ambiental. 1991. Avaliação da toxicidade crônica utilizando Ceriodapnhia dubia Richard, 1864 (Cladocera, Crustacea). São Paulo-SP.

CONAB. 2011. disponível em http: www.conab.gov.br. Acesso em 10 outubro de 2011.

FERNANDEZ-ALBA, A.R.; HERNANDO, M.D.; PIEDRA, L.; CHISTI, Y. 2002. Toxicity evaluation of single and mixed antifouling biocides measured with acute toxicity bioassays. Analytica Chimica Acta 456. 303-312.

FORGET-LERAY, J.; LANDRIAU, I.; MINIER, C.; LEBOULENGER, F. 2005. Impact of endocrine toxicants on survival, development, and reproduction of the estuarine copepod Eurytemora affinis (Poppe). Ecotoxicology and Environmental Safety, 60: 288-294.

FREITAS, E.C.; ROCHA, O.; 2012. Acute and chronic effects of atrazine and sodium dodecyl sulfate on the tropical freshwater cladoceran Pseudosida ramosa. Ecotoxicology 21: 1347-1357.

GIACOMAZZI, S.; COCHET, N.; 2004. Environmental impact of diuron transformation: a review. Chemosphere 56. 1021-1032.

HAMILTON, M.; RUSSO, R.C.; THURFTON, R.B. 1977. Trimmed Spearman-Karber method forestimating median lethal concentrations in toxicity biossays. EnvironmentalScience Technology, 11(7): 714-719.

Indicadores de desenvolvimento sustentável Brasil (IBGE). 2010. Disponível em http: www.ibge.gov.br/home/geociencias/recursosnaturais/ids /ids. Acesso em 20 outubro de 2011.

MBL Aquaculture (2005). Disponível em http://www.mblaquaculture.com/content/organisms/da phnids.php. Acesso em 20 outubro de 2011

MA, J.; WANG, S.; WANG, P.; MA, L.; CHEN, X.; 2006. Toxicity assessment of $\mathbf{4 0}$ herbicides to the green alga Raphidocelis subcapitata. Ecotox. And Environm. Safety 63. 456-462.

MONTAgUe, B. 2000. Oneliner Pesticide Toxicity Database. USEPA.

NEBEKER, A.V. \& SCHUYTEMA, G.S.. 1998. Chronic effects of the herbicide diuron on freshwater cladocerans, amphipods, midges, minnows, and snails. Arch. Environ. Contam. Toxicol. 35. 441-446.
NEUWOEHNER, J.; JUNGHANS, M.; KOLLER, M.; ESCHER, B.I.. 2008. QSAR analysis and specific endpoints for classifying the physiological modes of action of biocides in synchronous green algae. Aquat. Toxicol. 90. 8-18.

PASCHOALATO, C. F. P. R.; SILVA, B. M.; SOUZA, M. B.; BOLDRIN, T. R.; ROCHA, R. H.. 2013. Método Alternativo para contagem de algas utilizadas como alimento em ensaios de ecotoxicidade. 27을 Congresso Brasileiro de Engenharia Sanitária e Ambiental, I-356 15 a 19 setembro 2013 Goiânia- GO

RAND, G.M.; WELL, P.G.; McCARTHY, L.S. 1995. Introduction to aquatic toxicolohy: effects, evironmental fate, and risk assessment. $2^{\circ}$ edition. Taylor \& Francis.

RONCO, A.; BÁEZ, M.C.D.; GRANADOS, Y.P. 2004. Ensayos Toxicológicos y Métodos de Evaluación de Calidad de Aguas- Estandarización, Intercalibración, resultados y Aplicaciones. In: Morales, G.C. (ed). Centro Internacional de Investigaciones para el Desarrollo, Ottawa.

SANCHEZ-BAYO, F. 2006. Comparative acute toxicity of organic pollutants and reference values crustaceans, I. Branchiopoda, Copepoda and Ostracoda. Environmental Pollution. Vol. 139. 385-420p.

SANTOS, M. A. P. F.; VICENSOTTI, J.; MONTEIROR. T. R. 2007. Sensitivity of Four Test Organisms (Chironomus xanthus, Daphnia magna, Hydra attenuata and Pseudokirchneriella subcapitata) to $\mathrm{NaCl}$ : an Alternative Reference Toxicant. Journal of the Brazilian Society of Ecotoxicology. v 2, $n^{\circ} 3,229-236 p$.

SILVA, B.M.; RAVANELI, M.A.C.; PASCHOALATO, C.F.P.R.; 2010. Toxicidade aguda dos herbicidas diuron e hexazinona à Danio Rerio. Pesticidas: r. ecotoxicol. $e$ meio ambiente, Curitiba, v. 20, p. 17-28.

SILVA, J.D; COSTA, R.H.R.; MATIAS, W.G; JR CASTILHO, A.B.; 2012. Avaliação da Toxicidade de Lixiviados de Aterro Sanitário em Sistema de Lagoas de Estabilização com Testes de Toxicidade Aguda (Daphnia magna). São Paulo. Revista DAE, N.189, p.4049.

TATARAZAKO, N., ODA, S., WATANABE, H., MORITA, M. \& IGUSHI, T. 2003. Juvenile hormone agonists affect the occurrence of male Daphnia. Chemosphere, 53: 827-833.

USEPA - UNITED STATES EVIRONMENTAL PROTECTION AGENCY. Reregistration Eligibility Decision (RED) Hexazinone. 1997. WASHINGTON, D.C. 20460 
USEPA - UNITED STATES ENVIRONMENTAL PROTECTION AGENCY. 2001. Streamlined water effect ratio procedure for discharges of copper. Washington, D.C.41p.

USEPA -UNITED STATES EVIRONMENTAL PROTECTION AGENCY. 2003. Reregistration eligibility decision (RED) for Diuron. WASHINGTON, D.C. 20460. September 30, 2003

UNICA. Disponível em http: www.unicadata.com.br. Acessado em outubro de 2013.

WAN, M.T.; WATTS, R.G.; MOUL, D.J.. 1988. Evaluation of the acute toxicity to juvenile Pacific salmonids of hexazinone and its formulated products: Pronone 10G Velpar L, and their carriers. Bull. Environ. Contam. Toxicol., 41: 609-616.

WILLIAMSON, P. 1980. Variables affeecting body burdens of lead, zinc and cadmium in a roadside population of the snail Cepaea hortensis. O ecologia, 44: 213-220. 